\title{
Abbreviations and symbols
}

This appendix contains a list of abbreviations and symbols that are used in this volume. When referring to a chapter or section of the other volumes in this series, the following notational convention is used; otherwise the volumes are referred to with their titles.

A+section\# A3.2 refers to Section 3.2. in Huba Bartos ed. (to appear). Adjectival Phrases.

C+section\# C3.2 refers to Section 3.2. in Zoltán Bánréti ed. (to appear). Coordination and Ellipsis.

E+section\# E3.2 refers to Section 3.2. in Zsuzsanna Gécseg ed. (to appear). Finite Embedding.

F+section\# F3.2 refers to Section 3.2. in Tibor Laczkó \& Gábor Alberti eds. (to appear). Non-Finite and Semi-Finite Verb Phrases.

M+section\# M3.2 refers to Section 3.2. in Balázs Surányi ed. (to appear). Sentence Structure.

N+section\# N3.2 refers to Section 3.2 in Gábor Alberti and Tibor Laczkó eds. (2018) Nouns and Noun Phrases

V+section\# V3.2 refers to Section 3.2. in Károly Bibok ed. (to appear). Verb Phrases in General and Finite Verb Phrases.

\begin{tabular}{|c|c|c|c|}
\hline \multicolumn{4}{|c|}{$s$ used in both the ma } \\
\hline AP & Adjectival Phrase & NumP & Numeral Phrase \\
\hline $\mathrm{CP}$ & Complementizer Phrase & PP & Postpositional Phrase \\
\hline CTopic & Contrastive Topic & PredP & Predicative Phrase \\
\hline DP & Determiner Phrase & VM & Verb Modifier \\
\hline NP & Noun Phrase & VP & Verb Phrase \\
\hline
\end{tabular}

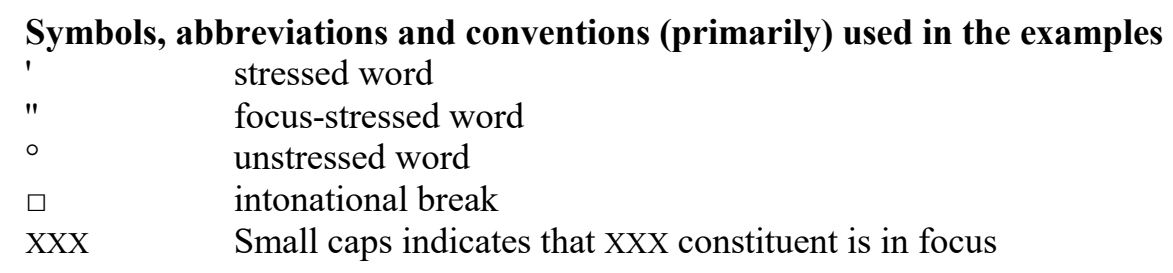




\section{Abbreviations used in the glosses of examples}

$\begin{array}{llll}1 / 2 / 3 & 1^{\text {st }}, 2^{\text {nd }}, 3^{\text {rd }} \text { person } & \text { Iter } & \text { Iterative suffix } \\ \text { Abl } & \text { Ablative } & \text { Loc } & \text { Obsolete locative case } \\ \text { Acc } & \text { Accusative } & \text { Min } & \text { Minimizing particle } \\ \text { Ade } & \text { Adessive } & \text { Mod } & \text { Modality suffix } \\ \text { Adj } & \text { Adjectivalizer } & \text { Mult } & \text { Multiplicative suffix } \\ \text { Adv } & \text { Adverbial suffix } & \text { Nmn } & \text { Nominalizer } \\ \text { All } & \text { Allative } & \text { Nom } & \text { Nominative } \\ \text { Attr } & \text { Attributivizer } & \text { Part } & \text { Participle } \\ \text { Car } & \text { Caritive suffix } & \text { Past } & \text { Past Tense }(-t) \\ \text { Cau } & \text { Causal(-final) } & \text { Perf } & \text { Perfectivizing } \\ \text { Caus } & \text { Causative } & & \text { verbal particle } \\ & \text { derivational suffix } & \text { Pl } & \text { Plural } \\ \text { Cl } & \text { Classifier } & \text { Poss } & \text { Possessed } \\ \text { Comp } & \text { Comparative } & \text { Posr } & \text { Possessor }(-e ́) \\ \text { Cond } & \text { Conditional } & \text { Pred } & \text { Predicate } \\ \text { Dat } & \text { Dative } & \text { Prox } & \text { Proximal } \\ \text { DefObj } & \text { Definite object } & \text { Quant } & \text { Quantifier } \\ \text { Del } & \text { Delative } & \text { QPart } & \text { Question particle }(-e) \\ \text { Dim } & \text { Diminutive } & \text { Rec } & \text { Reciprocal suffix } \\ \text { Dist } & \text { Distributive suffix } & \text { Sg } & \text { Singular } \\ \text { Dst } & \text { Distal } & \text { Sprl } & \text { Superlative prefix } \\ \text { Ela } & \text { Elative } & \text { Soc } & \text { Sociative suffix } \\ \text { Emph } & \text { Emphatic particle } & \text { Sub } & \text { Sublative } \\ \text { FoE } & \text { Essive-Formal } & \text { Subj } & \text { Subjunctive } \\ \text { For } & \text { Formal suffix } & \text { Sup } & \text { Superessive } \\ \text { Fut } & \text { Future } & \text { Ter } & \text { Terminative } \\ \text { Ill } & \text { Illative } & \text { Tmp } & \text { Temporal suffix } \\ \text { Ine } & \text { Inessive } & \text { TrE } & \text { Translative(-essive) } \\ \text { Inf } & \text { Infinitive } & \text { Vrb } & \text { Verbalizer } \\ \text { Ins } & \text { Instrumental } & & \\ & & & \end{array}$




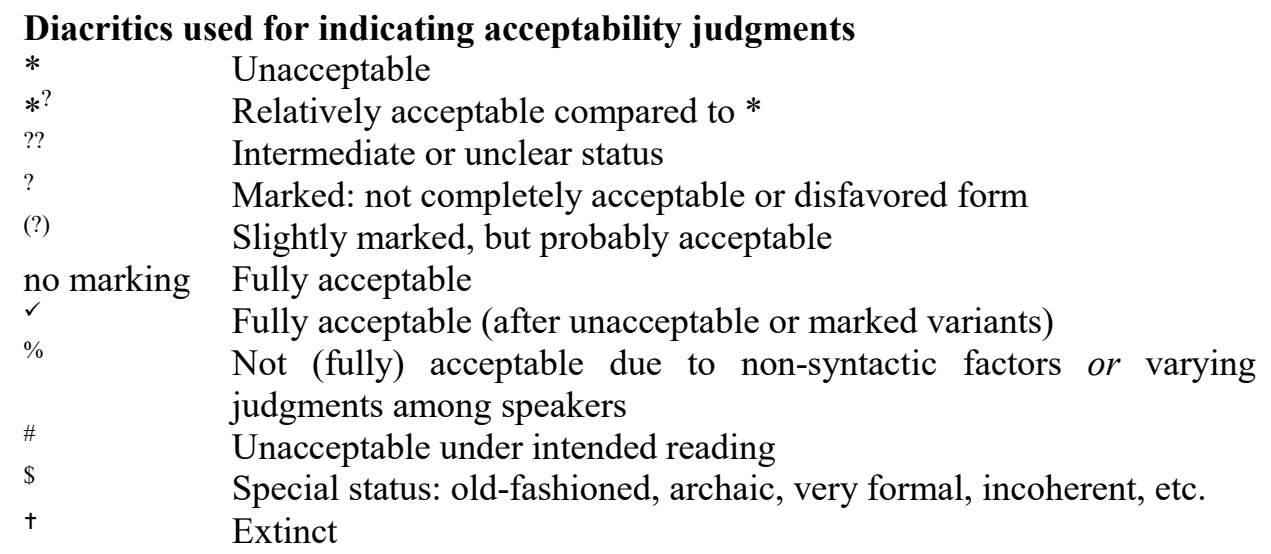

\section{Other conventions}

$\mathrm{xx} / \mathrm{yy}$

$*_{x x} / y y$

$\mathrm{xx} /$ *yy

$[\mathrm{y} \ldots \mathrm{z}]$

$\mathrm{xx} /[\mathrm{y} \ldots \mathrm{z}]$

Acceptable both with xx and with yy

Unacceptable with $\mathrm{xx}$, but acceptable with yy

Acceptable with $\mathrm{xx}$, but unacceptable with yy

A unit (but not necessarily a constituent) consisting of more than one word

$(\mathrm{xx})$

$*(\mathrm{xx})$

$(* x x)$

$(\mathrm{xx}) \ldots(\mathrm{xx})$

$\mathrm{XX}_{\mathrm{i}} \ldots \mathrm{YY}_{\mathrm{i}}$

$\mathrm{XX}_{\mathrm{i}} \ldots \mathrm{YY}_{\mathrm{j}}$

$\mathrm{XX}_{* \mathrm{i} / \mathrm{j}}$

$\mathrm{XX}_{\mathrm{i} / *_{\mathrm{j}}}$

$[\mathrm{XP} \cdots]$
Acceptable both with $\mathrm{xx}$, which is a word, and with [y ... z], which is a unit (but not necessarily a constituent) consisting of more than one word

Acceptable both with and without $\mathrm{xx}$

Acceptable with, but unacceptable without $\mathrm{xx}$

Acceptable without, but unacceptable with $\mathrm{xx}$

Alternative placement of $\mathrm{xx}$ in an example

Coindexing indicates coreference

Counter-indexing indicates disjoint reference

Unacceptable with index $i$, acceptable with index $j$

Unacceptable with index $j$, acceptable with index $i$

Constituent brackets of a constituent XP 
